\title{
Thiamine pyrophosphate improved vascular complications of diabetes in rats with type 2 diabetes by reducing glycation, oxidative stress, and inflammation markers
}

\author{
Sina Mahdavifard*1(D), Manochehr Nakhjavani² \\ Received: 30 Jun 2018 \\ Published: 13 May 2020
}

\section{Abstract}

Background: Thiamine deficiency contributes to hyperglycemia and diabetes complications. Thus, in this study, the effect of thiamine pyrophosphate (TPP) on the in vivo and in vitro formation of glycation, oxidative stress, and inflammatory markers (the main contributors of vascular diabetes complications) was examined in type 2 diabetes rat model.

Methods: Type 2 diabetes was induced in rats with a combination of streptozotocin and nicotinamide $(55+200 \mathrm{mg} / \mathrm{kg})$. Two groups of rats, healthy and diabetic, were treated with $0.1 \%$ TPP in drinking water daily for 3 months and the 2 others received water only. The glucose, insulin, early to end glycation products, the activity of glyoxalase system, lipid profile, LDL oxidation markers, inflammatory markers, creatinine in the serum, and proteinuria in the urine of all rats were determined. Moreover, albumin and LDL were incubated with glucose in the presence and absence of TPP, and the samples were investigated for glycation and oxidation products. Different variables in all 4 groups were compared with multiple analysis of variance (MANOVA-Tukey) test using SPSS version 16. Significance level was set at $\mathrm{p}<0.05$.

Results: TPP decreased the formation of diverse glycation and oxidation products in both in vivo (glycated LDL $=144.50 \pm 3.48$ and oxidized $\mathrm{LDL}=54.08 \pm 2.67 \mu \mathrm{mol} / \mathrm{l}$ ) and in vitro (glycated $\mathrm{LDL}=107.00 \pm 2.82$ and oxidized $\mathrm{LDL}=50.83 \pm 1.22 \mu \mathrm{mol} / \mathrm{l}$ ). In addition, the vitamin reduced fasting blood sugar (9.23 \pm 0.29$)$, insulin resistance $(9.10 \pm 0.50)$, tumor necrosis factor- $\alpha$ (285.43 \pm 15.97$)$, interleukin-6 (257.65 \pm 13.06$)$, and improved the lipid profile, the activity of Glo system (Glo-I $=31.65 \pm 1.06$ and Glo-II $=27.01 \pm 0.90 \mathrm{U} / \mathrm{mL}) \mathrm{and} \mathrm{renal}$ function in the diabetic rat $(\mathrm{p}<0.001)$.

Conclusion: TPP decreased the major risk factors for diabetic complications and corrected the alternations of glucose and lipid metabolism in type 2 diabetic rats; thus, it is recommended for diabetes treatment.

Keywords: Type 2 diabetes, Glycation, Oxidative stress, Inflammation, Streptozotocin, Diabetic vascular complications, Glyoxalase system, Proteinuria

Conflicts of Interest: None declared

Funding: Ardabil University of Medical Sciences

*This work has been published under CC BY-NC-SA 1.0 license.

Copyright $($ Iran University of Medical Sciences

Cite this article as: Mahdavifard S, Nakhjavani M. Thiamine pyrophosphate improved vascular complications of diabetes in rats with type 2 diabetes by reducing glycation, oxidative stress, and inflammation markers. Med J Islam Repub Iran. 2020 (13 May);34:47. https://doi.org/10.47176/mjiri.34.47

\section{Introduction}

Type 2 diabetes mellitus is one of the most common diseases in the world that causes serious health and economic

Corresponding author: Dr Sina Mahdavifard, s.mahdavifard@arums.ac.ir

1. Department of Clinical Biochemistry, Faculty of Medical Sciences, Ardabil University of Medical Sciences, Ardabil, Iran

2. Endocrine Division, Vali-asr Hospital, Tehran University of Medical Sciences, Tehran, Iran challenges. Hyperglycemia causes vascular diabetes complications owing to the induction of glycation, oxidative $\uparrow$ What is "already known" in this topic:

Hyperglycemia causes vascular diabetes complications owing to the induction of glycation, oxidative stress, and inflammation. Moreover, atherosclerosis and nephropathy are the main cause of death in these patients. thiamine deficiency leads to hyperglycemia and diabetes complications.

\section{$\rightarrow$ What this article adds:}

We suggested that thiamine pyrophosphate as an active form of the vitamin reduces glycation, oxidative stress, and inflammation markers as cardinal risk factors of vascular complications. Further, we proposed the vitamin improved activity of glyoxalase system, renal function and insulin sensitivity. 
stress, and inflammation (1). Moreover, atherosclerosis and nephropathy are the main causes of death in these patients (2).

Glycation products are the cardinal mediators of insulin resistance, $\beta$ cell failure, and diabetes disorders (3). Glycated LDL (g-LDL) and its oxidized form (Ox-LDL) contribute to the initiation and progression of atherosclerosis (4). The production of toxic carbonyl active species, such as glioxal (GO) and methylglyoxal (MGO), which is one of the consequences of oxidation of lipids and sugars, leads to carbonyl stress. Furthermore, this phenomenon is responsible for the activation of a series of inflammatory responses leading to accelerated vascular damage in diabetes (5). Glyoxalase system is the most potent enzymatic system for the prevention and reduction of carbonyl stress (6).

Thiamine is an antioxidant and a vital cofactor in the carbohydrate metabolism. Also, thiamine deficiency leads to hyperglycemia and diabetes complications (7). In this study, the effect of thiamine pyrophosphate (TPP) on glycemic, oxidative and inflammatory stress, as major risk factors for vascular disorders in diabetic rats, was studied. Also, the effect of treatment on the production of low glycemic and oxidized albumin and lipoprotein products in vitro was also investigated.

\section{Methods}

\section{Materials}

All materials were in analytical grade and purchased from Sigma or Merck Chemical Companies.

\section{In vivo studies}

\section{Animal model of type 2 diabetes}

Eight weeks old male Wistar rats, weighing $180 \pm 15$, were purchased from the Pasteur Institute, Karaj, Iran. Animals were housed under controlled temperature conditions with a 12-hour light and 12-hour dark cycle, with free access to food and water. After 2 weeks, they were divided into 2 main groups. After a 12-hour fasting, type 2 diabetes was induced in group 1 by single intraperitoneally (i.p.) injection nicotinamide $(210 \mathrm{mg} / \mathrm{kg}$ body weight in the $\mathrm{Na}-$ citrate buffer, $\mathrm{pH} 4.5)$ and streptozotocin $(55 \mathrm{mg} / \mathrm{kg}$ body weight in the citrate buffer, 15 min later) (8).

After 3 days, animals with fasting blood sugar of (FBS) $>11 \mathrm{mmol} / \mathrm{L}$ were considered diabetic. Then, each of the 2 main (diabetic and normal) groups was divided into 2 subgroups, with 10 rats in each: subgroup 1: D (for the diabetic group) and $\mathrm{N}$ (for the control group), with no more treatments; subgroups 2: D (TPP) and N (TPP) received $0.1 \%$ thiamine pyrophosphate (TPP) in drinking water daily for 3 months. The dose of thiamine was selected according to the literature (9) and the authors' experience.

All groups fed on a standard chow diet. The experimental protocol was approved by the Animal Ethical Committee in accordance with the guidelines for the care and use of laboratory animals prepared by Ardabil University of Medical Sciences.

At the end of the experiment, after a 16-hour fast, rats were anesthetized with an i.p injection of ketamine - xylosine $(90+10 \mathrm{mg} / \mathrm{kg}$ body mass). Then, a blood sample was collected from their heart and transferred into the test tubes with and without EDTA. Serum samples were prepared by 15 -minute centrifugation of blood at $5000 \times \mathrm{g}$ and were stored at $-70^{\circ} \mathrm{C}$ for measurements. Their kidneys were dissected and weighted promptly. The kidney weight index was calculated by dividing kidney weight to rats' body weight.

\section{Determining biochemical parameters}

Fasting blood sugar (FBS), triglyceride (TG), total cholesterol (TC), LDL, HDL, serum creatinine (Cr), and protein excretion in urine (PU) were measured by photometric methods. Moreover, the atherogenic index was computed with LDL/HDL ratio. The serum insulin level was determined by the enzyme-linked immunosorbent assay (ELISA) method using a rat insulin kit (Mercodia, Uppsala, Sweden) and HOMA-IR (homeostasis model assessment of insulin resistance) was calculated as explained previously (4).

\section{Determining glycated products}

Glycated albumin (g-Alb) was quantified by a colorimetric method using nitroblue tetrazolium chloride (10). Glycated LDL was determined by the reaction of extracting LDL with oxalic acid and thiobarbituric acid to yield the hydroxymethylfurfuraldehyde chromogen $(11,12)$. Glyoxal (GO) and methylglyoxal (MGO) were assayed by a reverse phase HPLC (10). The fluorescent AGEs were determined in the serum of rats (4).

\section{Determining oxidative stress markers}

Conjugated dienes (CD) in LDL lipids, as early products of LDL oxidation, was analyzed spectrometrically at 234 $\mathrm{nm}$. The molar extinction coefficient of CD is $2.95 \times 10^{4}$ $\mathrm{M}^{-1}$ and the results were presented as $\mu \mathrm{M}$. Fluorescence intensity of end oxidation product of LDL, named oxidation fluorescent product (OFP), was recorded at the emission maximum $430 \mathrm{~nm}$ upon excitation at $360 \mathrm{~nm}$ (10).

Advanced oxidation protein products (AOPP) was determined based on the spectrophotometric detection, according to the method of Witko-Sarsat et al, as described in our recent papers $(4,13)$. Levels of malondialdehyde (MDA) and glutathione (GSH) were determined with a commercial assay kit (Cayman Chemical Co., Ann Arbor, MI).

\section{Determining inflammatory markers}

Tumor necrosis factor- $\alpha$ (TNF- $\alpha$ ) and interleukin-6 (IL6 ) were determined using ELISA kits (Immunotech, France).

\section{Enzymatic assay}

The activity of GLO-1 in hemolysate was determined by measuring the initial rate of S-D-lactoylglutathione formation, while the rate of its hydrolysis was a measure of the activity of GLO-2 in all samples and expressed as unit $/ \mathrm{mL}$ $(10,14)$.

\section{In vitro albumin and $L D L$ glycation and oxidation Albumin extraction and glycation}

Rat serum albumin (RSA) was extracted and incubated with glucose in the absence and presence of TPP (100 
$\mathrm{mg} / \mathrm{L})$. The extraction and incubation procedures were similar to what was explained previously (4).

\section{Determining various glycated products of albumin}

All measurements were done at least 3 times, and in case of incompatibility, the experiment was repeated. The gAlb, MGO, GO, and fluorescent AGEs in the tubes containing the reaction mixtures were measured using the methods explained in the in vivo section.

\section{Purification and formation of glyco-oxidation products of $L D L$}

LDL was isolated with heparin and incubated in the presence and absence of glucose and TPP (100 mg/L). The complete procedure of the study was similar to that we explained before in this article (4).

\section{Determining glycated and oxidized LDL products}

The glycated and oxidized LDL products were measured using the methods explained in the in vivo section.

\section{Statistical analysis}

All data were expressed as mean \pm S.D (standard deviations). Different variables in all 4 groups were compared with one-way multiple analysis of variance (MANOVATukey) test using SPSS version 16. Significance level was set at $\mathrm{p}<0.05$.

\section{Results}

\section{The results of the in vivo experiment}

The levels of fasting blood sugar, insulin, HOMA-IR, lipid profile, and kidney function tests in the rat groups are represented in Table 1. FBS, HOMA index, as a marker of insulin resistance, TG, TC, LDL, and the atherogenic index increased significantly in the diabetic rats compared to the healthy group (Table 1). Thiamine pyrophosphate significantly reduced the parameters in diabetic rats. The treatment decreased the levels of TG, TC, and the atherogenic

Table 1. The effect of thiamine pyrophosphate (TPP) on fasting blood sugar, insulin, HOMA-IR, and glycation products of the healthy and diabetic rats

\begin{tabular}{|c|c|c|c|c|c|}
\hline \multirow[t]{2}{*}{ Variable } & \multicolumn{4}{|c|}{ Groups } & \multirow{2}{*}{$\frac{\mathrm{p}}{<0.001}$} \\
\hline & $\mathrm{N}$ & $\mathrm{N}(\mathrm{PLP}+\mathrm{THC})$ & $\mathrm{D}$ & $\mathrm{D}(\mathrm{PLP}+\mathrm{THC})$ & \\
\hline Blood sugar $(\mathrm{mmol} / \mathrm{L})$ & $4.50 \pm 0.27$ & $4.45 \pm 0.27$ & $15.73 \pm 0.46^{\mathrm{N}}$ & $9.23 \pm 0.29^{\mathrm{N}, \mathrm{D}}$ & $<0.001$ \\
\hline Insulin $(\mu \mathrm{U} / \mathrm{mL})$ & $17.04 \pm 0.90$ & $16.97 \pm 0.87$ & $13.03 \pm 0.71^{\mathrm{N}}$ & $11.72 \pm 0.34^{\mathrm{N}, \mathrm{D}}$ & $<0.001$ \\
\hline HOMA-IR & $3.40 \pm 0.427$ & $3.35 \pm 0.24$ & $9.10 \pm 0.50^{\mathrm{N}}$ & $4.80 \pm 0.20^{\mathrm{N}, \mathrm{D}}$ & $<0.001$ \\
\hline Triglyceride (mmol/L) & $0.92 \pm 0.02$ & $0.88 \pm 0.02^{\mathrm{N}, \mathrm{D}}$ & $3.10 \pm 0.16^{\mathrm{N}}$ & $1.81 \pm 0.08^{\mathrm{N}, \mathrm{D}}$ & $<0.001$ \\
\hline Total cholesterol $(\mathrm{mmol} / \mathrm{L})$ & $2.66 \pm 0.07$ & $2.35 \pm 0.08^{\mathrm{N}, \mathrm{D}}$ & $4.33 \pm 0.24^{\mathrm{N}}$ & $3.45 \pm 0.18^{\mathrm{N}, \mathrm{D}}$ & $<0.001$ \\
\hline $\mathrm{HDL}(\mathrm{mmol} / \mathrm{L})$ & $1.31 \pm 0.06$ & $1.27 \pm 0.05$ & $0.48 \pm 0.01^{\mathrm{N}}$ & $1.00 \pm 0.03^{\mathrm{N}, \mathrm{D}}$ & $<0.001$ \\
\hline $\mathrm{LDL}(\mathrm{mmol} / \mathrm{L})$ & $0.58 \pm 0.01$ & $0.53 \pm 0.02$ & $2.61 \pm 0.24^{\mathrm{N}}$ & $1.53 \pm 0.16^{\mathrm{N}, \mathrm{D}}$ & $<0.001$ \\
\hline $\mathrm{HDL} / \mathrm{LDL}$ & $0.44 \pm 0.01$ & $0.41 \pm 0.01^{\mathrm{N}, \mathrm{D}}$ & $5.43 \pm 0.49^{\mathrm{N}}$ & $1.53 \pm 0.11^{\mathrm{N}, \mathrm{D}}$ & $<0.001$ \\
\hline Creatinine $(\mu \mathrm{mol} / \mathrm{L})$ & $59.65 \pm 3.87$ & $65.70 \pm 4.29$ & $105.73 \pm 6.53^{\mathrm{N}}$ & $83.08 \pm 5.09^{\mathrm{N}, \mathrm{D}}$ & $<0.001$ \\
\hline Proteinuria (mg/24 h) & $11.73 \pm 0.95$ & $9.35 \pm 0.81$ & $320.62 \pm 17.63^{\mathrm{N}}$ & $65.25 \pm 3.92^{\mathrm{N}, \mathrm{D}}$ & $<0.001$ \\
\hline Kidney weight index & $0.77 \pm 0.05$ & $0.75 \pm 0.04$ & $1.350 .77 \pm 0.71^{\mathrm{N}}$ & $0.81 \pm 0.05^{\mathrm{D}}$ & $<0.001$ \\
\hline
\end{tabular}

$\mathrm{N}$ indicates significance of data comparing group $\mathrm{N}$ (normal) with other groups

${ }^{\mathrm{D}}$ indicates significance of data comparing group D (diabetic) with other groups

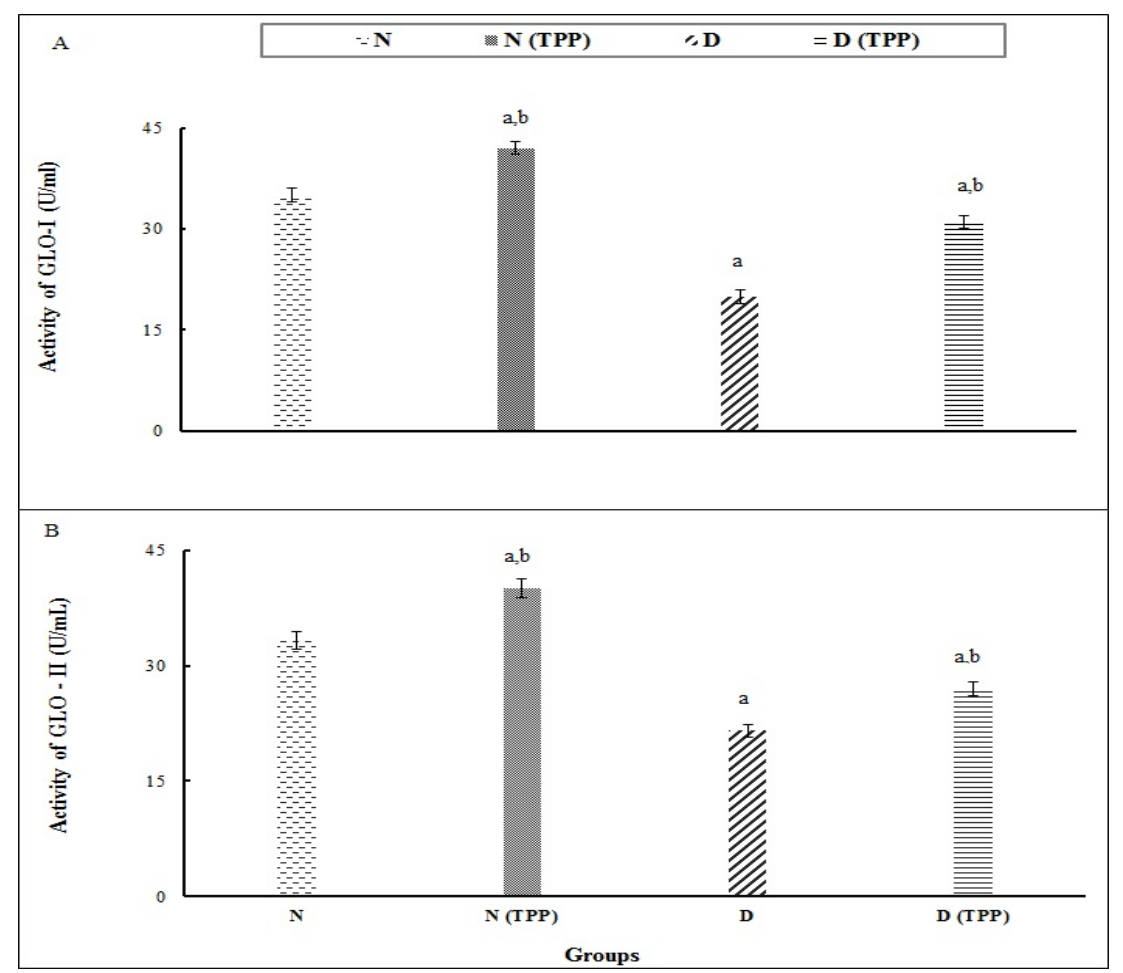

Fig. 1. Effect of thiamine pyrophosphate on the activity of glyoxalase system in healthy and diabetic rats: (A) Activity of glyoxalase-I; (B) Activity of glyoxalase-II. a indicates significance of data comparing the healthy group $(\mathrm{N})$ with other groups $(\mathrm{p}<0.001)$.

${ }^{b}$ indicates significance of data comparing group diabetic (D) with other groups ( $\left.<<0.001\right)$. 
index in the treated healthy rats compared to untreated rats $(\mathrm{p}<0.001)$.

Glyoxalase-I and II activities in treated and untreated rat groups are presented in Figure 1. Diabetes induction in rats decreased the activity of Glo system, but the vitamin induced the activity of Glo-I and Glo-II in the healthy and diabetic rats $(\mathrm{p}<0.001)$.

Comparison of the effect of the treatment on glycation ( $\mathrm{g}$ Alb, g-LDL, GO, MGO, and AGES), oxidative stress (early and end oxidation products of LDL, AOPP, MDA, and GSH), and inflammatory (TNF- $\alpha$ and IL-6) markers in the named groups is represented in Table 2. Levels of glycation, oxidative stress, and inflammatory markers decreased in the treated diabetic rats than in the untreated, but GSH level was higher in the treated than in the untreated diabetic rats $(p<0001)$. Furthermore, the levels of the glycation and inflammatory markers were lower in the healthy rats compared to the untreated rats $(\mathrm{p}<0.001)$.

\section{The results of the in vitro study}

The effects of TPP on the in vitro formation of various glycation products (early to end) of RSA and LDL glycation and oxidation (early and end) products are presented in Table 3. The vitamin diminished the levels of early ( $\mathrm{g}$ Alb), intermediate ( $\mathrm{GO}$ and $\mathrm{MG}$ ), and end products (AGEs) of glycation as well as LDL glycation and oxidation products.

\section{Discussion}

The effect of thiamine pyrophosphate on the activity of the glyoxalase system, various glycation products (early to end), oxidative stress markers, as early and end oxidation products of LDL, as well as advanced oxidation protein products (AOPP) has not been explored in rats with type 2 diabetes. In this study, thiamine pyrophosphate reduced various glycation, oxidative stress, and inflammatory markers, as risk factors of vascular diabetes complications, in rats with type 2 diabetes. Moreover, the treatment showed a beneficial effect on glucose metabolism, lipid profile, glyoxalase system activity, and kidney function. Also, in vitro inhibitory property of the vitamin on the diverse glycation and oxidation products of albumin and LDL was observed.

Diabetes and thiamine deficiency reduce the activity of the glutoxylase system. TPP decreased blood glucose along with improving insulin action in the diabetic rats. Insulin and HOMA-IR levels were, respectively, higher and lower $(p<0.001)$ in treated diabetic rats than in the untreated group (Table 1). Thiamine is necessary for insulin synthesis and secretion (15). In the thiamine-deficient state, glucose undergoes metabolism via alternate pathways, which can result in insulin resistance and complications (16). The glyoxalase system decreases dicarbonyl compounds and AGEs (17). Diabetes and deficiency of thiamine reduce the activity of Glo system. Moreover, thiamine deficiency is common in diabetes $(4,10)$. Various glycation products, including glycated albumin, dicarbonyl compounds, and AGEs, have been known as the sources of oxidative stress and inflammatory markers that induce insulin-resistance, hypertension, neurodegenerative disease, and diabetic vascular complications $(18,19)$. AGEs may interfere in the insulinsecretion (20). Prerhaps, AGEs were involved in the pathogenesis of insulin resistance and accelerated atherosclerosis in diabetes through the induction of oxidative stress and inflammation (21). In this study, TPP, as a potent

Table 2. Effect of thiamine pyrophosphate (TPP) on the lipid profile of healthy and diabetic rats

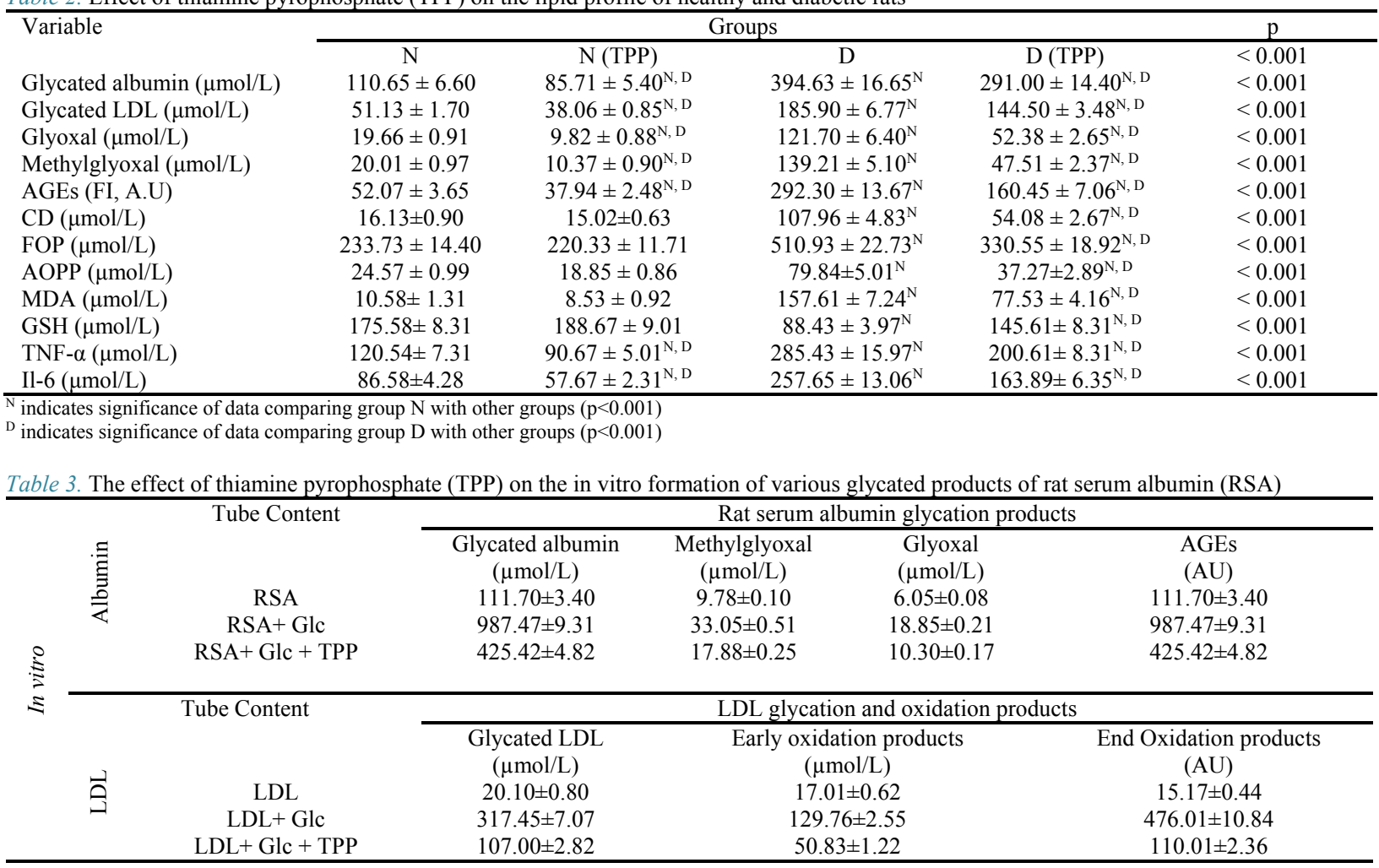


antiglycating compound, induced the activities of glyoxalase-1 and glyoxalase-2 (Fig. 1. A and 1.B) and decreased the early (g-Alb and g-LDL), intermediate (GO and MGO), and end glycation products (AGEs) in the treated healthy and diabetic rats (Table 2$)(\mathrm{p}<0.001)$. Further, the vitamin has in vitro antiglycating activity on diverse glycation products (Table 3). Based on the literature review, the effect of TPP on the Glo system activity in diabetes has not yet been investigated. The reducing effect of thiamine on the formation of AGEs in vitro and in vivo as well as MGO formation in the in vitro were presented $(22,23)$. However, the effects of thiamine on different glycation products in vivo and in vitro was investigated. Thiamine may reduce different glycation products with antioxidant property, dicarbonyl scavenger, and activation of the Glo system (24).

Oxidative stress and inflammation stimulate insulin resistance and diabetes complications (25). Thus, the treatment decreased insulin resistance and intercepted atherosclerosis over and above amended nephropathy in the diabetic rats because of reducing oxidative stress. Over and above the treatment decreased advanced oxidation protein products and malondialdehyde, along with the elevation of glutathione level. Further, it abstracted TNF- $\alpha$ and IL- 6 as inflammatory markers (Table 2). Previously, the anti-inflammatory effect of thiamine with reduction of cited inflammatory markers was reported in a chronic model of inflammation in the rat (26).

The rising levels of TG, LDL, and VLDL, along with a reduction of HDL, were observed in diabetic nephropathy, diabetic microalbuminuria, and thiamine deficiency (27, 28). The treatment had a positive effect on the lipid profile in the healthy and diabetic rats (Table 1). The treatment decreased TG, TC, and the atherogenic index in the healthy and diabetic rats but reduced LDL only in the diabetic rats $(p<0.001)$. Thiamine reduces lipogenesis in the liver and adipose tissue in glycemic condition (29).

Glycated LDL is more sensitive to oxidation than the nonglycated of LDL. Oxidized LDL has a principal role in the initiation and progression of atherosclerosis (30). Glycation and oxidation of LDL increase following glycemia, dyslipidemia, oxidative stress, and glycation $(31,32)$. The results of this study revealed an inhibitory effect of TPP on the glycation and oxidation of LDL in both conditions, in vivo $(p<0.001)$ and in vitro (Tables 2 and 3$)$ for the first time. The treatments that inhibit the formation of the end oxidation products of LDL can prevent the formation of any atheromatous lesions $(4,10)$.

Diabetic nephropathy (DN) contributes to renal failure. Diabetes induction elevated urinary protein excretion, serum creatinine, and kidney weight index in the rats (Table 1). Moreover, a negative correlation was found between kidney weight index and renal function (4). The vitamin reduced the levels of kidney function markers in the diabetic rats $(p<0.001)$. Furthermore, no difference was observed between kidney weight index in the treated diabetic rats and the healthy rats. Level of $\mathrm{g}-\mathrm{Alb}$ is a better predictor of $\mathrm{DN}$ progression than glycated hemoglobin in humans with type 2 diabetes (33). Moreover, AGEs have a cardinal role in the progression of vascular dysfunction due to induction of ox- idative stress and inflammation (34). Proinflammatory cytokines, as TNF- $\alpha$ and IL- 6 , contribute to renal lesions through cellular injury, alteration of the glomerular protein permeability barrier, and development of intracranial inflammatory damage which lead to diabetic nephropathy (35). TPP improved kidney dysfunction in diabetic rats by reducing glycation, protein, lipid oxidation, and inflammatory markers (Table 2). In accordance with the results of the present study, there is some evidence that indicate the increase in glycated albumin, MGO, and AGEs and the decrease in the activity of Glo-1 are associated with nephropathy $(4,10)$. In this study, for the first time, the healing effect of thiamine pyrophosphate on renal impairment in type 2 diabetic rats was reported.

\section{Conclusion}

Thiamine pyrophosphate improves vascular diabetes complications by the loweing effect on glycation, oxidative stress, and inflammation markers and by an advantageous effect on insulin action, the activity of glyoxalase system, and lipid profile in the diabetic rats.

\section{Acknowledgments}

The authors are thankful to Ardabil University of Medical Sciences

\section{Conflict of Interests}

The authors declare that they have no competing interests.

\section{References}

1. Kawanami D, Matoba K, Utsunomiya K. Signaling pathways in diabetic nephropathy. Histol Histopathol. 2016;31(10):1059-67.

2. De Nicola L, Gabbai FB, Liberti ME, Sagliocca A, Conte G, Minutolo R. Sodium/glucose cotransporter 2 inhibitors and prevention of diabetic nephropathy: Targeting the renal tubule in diabetes. Am J Kidney Dis. 2014;64:16-24.

3. Vlassara H, Uribarri J. Advanced glycation end products (AGE) and diabetes: Cause, effect, or both? Curr Diab Rep. 2014;14:1-17.

4. Mahdavifard S, Bathaie SZ, Nakhjavani M, Taghikhani M. The synergistic effect of antiglycating agents (MB-92) on inhibition of protein glycation, misfolding and diabetic complications in diabeticatherosclerotic rat. Eur J Med Chem. 2016;121:892-902.

5. Shayesteh R, Kamalinejad M, Adiban H, Kardan A, Keyhanfar F, Eskandari MR. Cytoprotective Effects of Pumpkin (Cucurbita Moschata) Fruit Extract against Oxidative Stress and Carbonyl Stress. Drug Res (Stuttg). 2017;67(10):576-82.

6. Maessen DE, Stehouwer CD, Schalkwijk CG. The role of methylglyoxal and the glyoxalase system in diabetes and other agerelated diseases. Clin Sci (Lond). 2015;128(12):839-61.

7. Gangolf M, Czerniecki J, Radermecker M, Detry O, Nisolle M, Jouan $\mathrm{C}$, et al. Thiamine status in humans and content of phosphorylated thiamine derivatives in biopsies and cultured cells. PLoS One. 2010;5(10):e13616.

8. Taha H, Arya A, Paydar M, Looi CY, Wong WF, Vasudeva Murthy $\mathrm{CR}$, et al. Upregulation of insulin secretion and downregulation of proinflammatory cytokines, oxidative stress and hyperglycemia in STZnicotinamide-induced type 2 diabetic rats by Pseuduvaria monticola bark extract. Food Chem Toxicol. 2014;66:295-306.

9. Rabbani N, Alam SS, Riaz S, Larkin J, Akhtar MW, Shafi T, et al. High-dose thiamine therapy for patients with type 2 diabetes and microalbuminuria: a randomised, double-blind placebo-controlled pilot study. Diabetologia. 2009;52(2):208-12.

10. Mahdavifard S, Bathaie S, Nakhjavani M, Heidarzadeh H. L-cysteine is a potent inhibitor of protein glycation on both albumin and LDL, and prevents the diabetic complications in diabetic-atherosclerotic rat. 
Food Res Int. 2014;62:909-16.

11. Schaefer EJ, Otokozawa S, Ai M. Limitations of direct methods and the reference method for measuring HDL and LDL cholesterol. Clin Chem. 2011;57(7):1081-3; author reply 3 .

12. Younis N, Charlton-Menys V, Sharma R, Soran H, Durrington PN. Glycation of LDL in non-diabetic people: Small dense LDL is preferentially glycated both in vivo and in vitro. Atherosclerosis. 2009;202(1):162-8.

13. Shahsavari Gh, Firozi M, Mahdavifard S, Judaki A, Birjandi M . Phototherapy motivates protein and lipid oxidation in jaundiced term and late term neonates. Caspian J Pediatr. 2017;3(2):248-52.

14. Peters AS, Wortmann M, Fleming TH, Nawroth PP, Bruckner T, Bockler D, et al. Effect of metformin treatment in patients with type 2 diabetes with respect to glyoxalase 1 activity in atherosclerotic lesions. Vasa. 2018;13:1-7.

15. Balakumar P, Rohilla A, Krishan P, Solairaj P, Thangathirupathi A. The multifaceted therapeutic potential of benfotiamine. Pharmacol Res. 2010;61(6):482-8

16. Alaei Shahmiri F SM, Zhao Y, Sherriff J. High-dose thiamine supplementation improves glucose tolerance in hyperglycemic individuals: a randomized, double-blind cross-over trial. Eur J Nutr. 2013;52(7):1821-4

17. Giacco F, Du X, D'Agati VD, Milne R, Sui G, Geoffrion M, et al. Knockdown of glyoxalase 1 mimics diabetic nephropathy in nondiabetic mice. Diabetes. 2014;63(1):291-9.

18. Sarkar P, Kar K, Mondal MC, Chakraborty I, Kar M. Elevated level of carbonyl compounds correlates with insulin resistance in type 2 diabetes. Ann Acad Med Singapore. 2010;39(12):909-4.

19. Fournet M, Bonte F, Desmouliere A. Glycation Damage: A Possible Hub for Major Pathophysiological Disorders and Aging. Aging Dis. 2018;9(5):880-900.

20. Okura T, Ueta E, Nakamura R, Fujioka Y, Sumi K, Matsumoto K, et al. High Serum Advanced Glycation End Products Are Associated with Decreased Insulin Secretion in Patients with Type 2 Diabetes: A Brief Report. J Diabetes Res. 2017;2017:5139750.

21. Yamagishi SI, Matsui T. Role of Hyperglycemia-Induced Advanced Glycation End Product (AGE) Accumulation in Atherosclerosis. Ann Vasc Dis. 2018;11(3):253-8.

22. Tarwadi KV, Agte VV. Effect of micronutrients on methylglyoxalmediated in vitro glycation of albumin. Biol Trace Elem Res. 2011;143(2):717-25.

23. Pearce K, Hatzinikolas A, Moran L, de Courten MPJ, Forbes J, Scheijen J, et al. Disparity in the micronutrient content of diets high or low in advanced glycation end products (AGEs) does not explain changes in insulin sensitivity. Int J Food Sci Nutr. 2017;68(8):1021-6.

24. Stratmann B, Engelbrecht B, Espelage BC, Klusmeier N, Tiemann J, Gawlowski $\mathrm{T}$, et al. Glyoxalase 1-knockdown in human aortic endothelial cells - effect on the proteome and endothelial function estimates. Sci Rep. 2016;6:37737.

25. Al-Trad B, Alkhateeb H, Alsmadi W, Al-Zoubi M. Eugenol ameliorates insulin resistance, oxidative stress and inflammation in high fat-diet/streptozotocin-induced diabetic rat. Life Sci. 2019;216:183-8.

26. Menezes RR GA, Rodrigues FF, Coura GME, Melo ISF, Brito AMS, Bertollo CM, Paulino TP, Rachid MA, Machado RR, Coelho MM. Thiamine and riboflavin inhibit production of cytokines and increase the anti-inflammatory activity of a corticosteroid in a chronic model of inflammation induced by complete Freund's adjuvant. Pharmacol Rep. 2017;69(5):1036-43.

27. Barter PJ. Lipoprotein metabolism and CKD: Overview. Clin Exp Nephrol. 2014;18:243-6.

28. Waheed P, Naveed AK, Ahmed T. Thiamine deficiency and its correlation with dyslipidaemia in diabetics with microalbuminuria. $\mathrm{J}$ Pak Med Assoc. 2013;63(340).

29. Gunanti IR, Marks GC, Al-Mamun A, Long KZ. Low serum vitamin B-12 and folate concentrations and low thiamin and riboflavin intakes are inversely associated with greater adiposity in Mexican American children. J Nutr. 2014;144(12):2027-33

30. Dos Santos MM, Prestes AS, de Macedo GT, Ecker A, Barcelos RP, Boligon AA, et al. Syzygium cumini leaf extract inhibits LDL oxidation, but does not protect the liproprotein from glycation. J Ethnopharmacol. 2018;210:69-79.

31. Steinberg D, Witztum JL. Oxidized low-density lipoprotein and atherosclerosis. Arterioscler Thromb Vasc Biol. 2010;30:2311-6.

32. Cai R, Chen S, Jiang S. [Chlorogenic acid inhibits non-enzymatic glycation and oxidation of low density lipoprotein]. Zhejiang Da Xue Xue Bao Yi Xue Ban. 2018;47(1):27-34.

33. Raghav A, Ahmad J, Noor S, Alam K, Mishra BK. Glycated albumin and the risk of chronic kidney disease in subjects with Type 2 Diabetes: A study in North Indian Population. Diabetes Metab Syndr. 2018;12(3):381-5.

34. Baradaran A, Nasri H, Rafieian-Kopaei M. Protection of renal tubular cells by antioxidants: current knowledge and new trends. Cell J. 2015;16(4):568-71.

35. Chu S, Hu L, Wang X, Sun S, Zhang T, Sun Z, et al. Xuezhikang ameliorates contrast media-induced nephropathy in rats via suppression of oxidative stress, inflammatory responses and apoptosis. Ren Fail. 2016;38(10):1717-25. 